Research Paper

\title{
Do Higher Radiation Doses with Concurrent Chemotherapy in the Definitive Treatment of Esophageal Cancer Improve Outcomes? A Meta-Analysis and Systematic Review
}

\author{
Linlin Xiao"\#, Brian G. Czito, Qingsong Pang ${ }^{3}$, Zhouguang Hui ${ }^{4}$, Shaowu Jing1, Baoen Shan ${ }^{*}$, Jun \\ Wang ${ }^{1 凶}$ \\ 1. Department of Radiotherapy, Fourth Hospital of Hebei Medical University, Shijiazhuang, Hebei, China \\ 2. Department of Radiation Oncology, Duke University, Durham, North Carolina, USA. \\ 3. Department of Radiotherapy, National Clinical Research Center for Cancer, Tianjin's Clinical Research Center for Cancer, Key Laboratory of Cancer \\ Prevention and Therapy, Tianjin Medical University Cancer Institute and Hospital, Tianjin, China. \\ 4. Department of Radiotherapy, Cancer Institute \& Hospital, Peking Union Medical College, \& Chinese Academy of Medical Sciences, Beijing, China. \\ \#: These authors contributed equally to this work. \\ *: These authors contributed equally to this work. \\ $\square$ Corresponding author: Dr. Jun Wang, wangjun0818@hebmu.edu.cn; Tel: 0311-86095752; Dr. Baoen Shan; baoenshan@hbydsy.com; Tel: 0311-86095287
}

(c) The author(s). This is an open access article distributed under the terms of the Creative Commons Attribution License (https://creativecommons.org/licenses/by/4.0/). See http://ivyspring.com/terms for full terms and conditions.

Received: 2020.01.31; Accepted: 2020.04.26; Published: 2020.05.18

\begin{abstract}
Background: To investigate the effects and safety profile of radiation dose escalation utilizing computerized tomography (CT) based radiotherapy techniques (including 3-Dimensional conformal radiotherapy, intensity-modulated radiotherapy and proton therapy) in the definitive treatment of patients with esophageal carcinoma (EC) with definitive concurrent chemoradiotherapy (dCCRT).

Methods: All relevant studies utilizing CT-based radiation planning, comparing high-dose ( $\geq 60 \mathrm{~Gy}$ ) versus standard-dose $(50.4 \mathrm{~Gy})$ radiation for patients with $\mathrm{EC}$ were analyzed for this meta-analysis.

Results: Eleven studies including 4946 patients met the inclusion criteria, with $96.5 \%$ of patients diagnosed with esophageal squamous cell carcinoma (ESCC). The high-dose group demonstrated a significant improvement in local-regional failure (LRF) (OR 2.199, 95\% Cl 1.487-3.253; $\mathrm{P}<0.001$ ), two-year local-regional control (LRC) (OR 0.478, 95\% Cl 0.309-0.740; $\mathrm{P}=0.001)$, two-year overall survival (OS) (HR 0.744, 95\% Cl 0.657-0.843; P<0.001) and five-year OS (HR 0.683, 95\% Cl 0.561-0.831; $\mathrm{P}<0.001)$ rates relative to the standard-dose group. In addition, there was no difference in grade $\geq 3$ radiation-related toxicities and treatment-related deaths between the groups.

Conclusion: Under the premise of controlling the rate of toxicities, doses of $\geq 60 \mathrm{~Gy}$ in CT-based dCCRT of ESCC patients might improve locoregional control and ultimate survival compared to the standard-dose dCCRT. While our review supports a dose-escalation approach in these patients, multiple ongoing randomized trial initial and final reports are awaited to evaluate the effectiveness of this strategy.
\end{abstract}

Key words: definitive concurrent chemoradiotherapy, dose escalation, esophageal cancer, esophageal squamous cell carcinoma, radiation dose

\section{Introduction}

Globally, esophageal cancer (EC) is one of the most common causes of cancer-related death [1, 2]. Collectively, the 5-year overall survival (OS) rate for all patients is $19 \%$ [2]. For non-operable, localized EC, definitive concurrent chemoradiotherapy (dCCRT) is usually the standard treatment approach. Currently, the dCCRT radiation dose recommended by National Comprehensive Cancer Network (NCCN) is 50.4 Gy 
[3], which is primarily based on results of the Radiation Therapy Oncology Group (RTOG) 85-01 and 94-05 randomized trials ${ }^{[4-6]}$. However, the optimal radiation dose in this scenario remains controversial. Many studies have demonstrated that local-regional failure (LRF) remains a common failure pattern for EC patients following dCCRT, most likely to occur within the original gross tumor volume (GTV), even in patients achieving clinical complete response (cCR) following treatment [7-11]. In addition, LRF rates of esophageal squamous cell carcinoma (ESCC) appear to be higher than that of esophageal adenocarcinoma (EAC) [12, 13]. Because patients with EAC generally have lower $\mathrm{CCR}$ rates with chemoradiation and are frequently considered for surgical resection following neoadjuvant treatment (with resultant high rates of local control), these patients have not been the primary focus of dose escalation studies. However, patients with ESCC often have additional comorbidities and generally achieve higher $\mathrm{CCR}$ rates with chemoradiation, leading to consideration of non-operative approaches. Therefore, the role of dose escalation is more relevant in patients with ESCC, particularly given that LRF rates are high following standard chemoradiation approaches.

Given the above, radiation dose escalation has been proposed as a technique to obtain higher local-regional control (LRC) and survival rates, notably in Asian countries [14-24]. In recent years, multiple investigators have carried out studies comparing the curative impact of high-dose versus standard-dose radiation treatments, although conclusions have been inconsistent [14-31]. Additionally, with advances in radiotherapy techniques, questions have been raised as to whether dose escalation utilizing computerized tomography (CT)-based radiotherapy approaches (including 3-Dimensional conformal radiotherapy (3D-CRT), intensity-modulated radiotherapy (IMRT), and proton therapy) could achieve improved outcomes with less toxicity compared to older approaches from the 2-D era. Based on the above, we undertook this meta-analysis to explore whether dose escalation utilizing CT-based radiotherapy techniques could benefit patients with EC receiving dCCRT.

\section{Methods}

Studies published prior to February 2020 comparing radiation dose and disease-related outcomes in nonoperable EC patients were included. Search keywords included "esophageal or oesophageal" and "carcinoma or cancer or neoplasm" and "chemoradiotherapy or chemoradiation or radiochemotherapy or irradiation or chemo-irradiation" and "dose or dose escalation or high dose". After retrieval, we manually filtered the articles by abstract and/or full text review. Inclusion criteria included: 1) CT-based radiotherapy techniques, such as 3-D, IMRT, or proton beam therapy, were utilized (articles that applied 2D radiotherapy techniques were excluded); 2) dCCRT was used (articles that reported sequential treatments, neoadjuvant or adjuvant CRT combined with surgery, palliative CRT or radiotherapy alone were excluded); 3) subject patients were stratified into high-dose ( $\geq 60$ Gy) and standard-dose (approximately 50 Gy) groups, with comparative data provided; 4) accurate statistical methods, valid data, and clear conclusions were given; 5) hazard ratio (HR) and 95\% confidence intervals (CI) were provided or could be calculated.

Outcome data included cCR rate, LRF rate, two-year LRC rate, two- and five-year OS, grade $\geq 3$ radiation-related toxicities and treatment-related deaths.

Case-control study evaluation guidelines were applied in order to evaluate the quality of each manuscript for the following criteria: 1) whether gender, age, and tumor location were clearly stated; 2 ) whether the comparability of the two groups was analyzed; 3) whether the statistical method was appropriate; 4) whether biases were discussed in the study. A point was assigned for each of these four items, with a total score of $\geq 3$ indicative of reliable quality. Two researchers independently reviewed the literature according to the unified quality standard, with results crosschecked. If there were some different opinions, a third researcher would be invited to solve the disagreement.

Data were analyzed using Stata version 11.0. Hazard ratio (HR), odds ratio (OR) and 95\% CI were used to measure effect size. A $Q$ test was applied to test for result heterogeneity. If $P>0.05$, the fixed effect model was used for statistical consolidation. If $\mathrm{P} \leq$ 0.05 , the random effect model was used. The combined effect size was tested utilizing the $\mathrm{z}$ test. Funnel plots were created to evaluate the risk of publication bias.

\section{Results}

\section{Literature search and study selection}

1351 articles were yielded initially. Ultimately, 1340 articles were excluded as outlined in in Figure 1. Eleven articles were selected for the final analysis, including nine retrospective studies and one prospective randomized study [14-19, 23, 25-28] (Table 1). There were 4946 EC patients included in the analyzed cohort, including 4775 ESCC patients, 142 EAC patients and 29 patients with other histology. 
Table 1. Basic characteristics of the included studies

\begin{tabular}{|c|c|c|c|c|c|c|c|c|c|}
\hline Author & Year & Nation & Study design & SCC/AC & TNM stage & Groups & Patients Number & Radiation dose $\mathrm{e}^{\#}$ & Category of RT type \\
\hline \multirow[t]{2}{*}{ He et al [14] } & 2014 & USA & Retrospective & $193 / 0$ & I-IV & Standard dose & 137 & Median: 50.4 Gy (41.4-50.4 Gy) & 3D-CRT, IMRT and Proton therapy \\
\hline & & & & & & High dose & 56 & Median: 60 Gy (52.2-66 Gy) & \\
\hline \multirow[t]{2}{*}{ Chen et al [15] } & 2016 & China & Retrospective* & $648 / 0$ & I-IV & Standard dose & 324 & $50-50.4 \mathrm{~Gy}$ & 3D-CRT and IMRT \\
\hline & & & & & & High dose & 324 & $\geq 60$ Gy & \\
\hline \multirow[t]{2}{*}{ Chang et al [16] } & 2017 & China & Retrospective & $2061 / 0$ & IA-IIIC & Standard dose & 1134 & Median: 50.4 Gy (45-59.4 Gy) & IMRT \\
\hline & & & & & & High dose & 927 & Median: 66.6 Gy (60-72 Gy) & \\
\hline \multirow[t]{2}{*}{ Kim et al [17] } & 2017 & Korea & Retrospective & $230 / 6$ & II-III & Standard dose & 120 & Median: 50.4 Gy (45-59.4 Gy) & 3D-CRT and IMRT \\
\hline & & & & & & High dose & 116 & Median: 63 Gy (60-66.6 Gy) & \\
\hline \multirow[t]{2}{*}{ Nayan et al [18] } & 2018 & India & Prospective & $28 / 0$ & II-III & Standard dose & 14 & $50.4 \mathrm{~Gy}$ & 3D-CRT and IMRT \\
\hline & & & & & & High dose & 14 & $64.8 \mathrm{~Gy}$ & \\
\hline \multirow[t]{2}{*}{ Ren et al [19] } & 2018 & China & Retrospective* & $380 / 0$ & I-IV & Standard dose & 190 & 50.4-54 Gy & 3D-CRT and IMRT \\
\hline & & & & & & High dose & 190 & $60 \mathrm{~Gy}$ & \\
\hline \multirow[t]{2}{*}{ Ke et al [23] } & 2018 & China & Retrospective & $84 / 0$ & I-III & Standard dose & 42 & Median: 49.5 Gy (44-50.4 Gy) & IMRT and Conformal Arc \\
\hline & & & & & & High dose & 42 & Median: 61.8 Gy (52.2-70 Gy) & \\
\hline \multirow[t]{2}{*}{ Welsh et al [25] } & 2016 & USA & Retrospective & $45 / 89$ & I-IV & Standard dose & 97 & $50.4 \mathrm{~Gy}$ & IMRT and Proton therapy \\
\hline & & & & & & High dose & 38 & Median: 63 Gy (58.8-63 Gy) & \\
\hline \multirow[t]{2}{*}{ Clavier et al [26] } & 2013 & France & Retrospective & $113 / 30$ & I-IV & Standard dose & 60 & Median: 50Gy (38-50.4 Gy) & 3D-CRT and IMRT \\
\hline & & & & & & High dose & 83 & Median: 66Gy (50.7-72 Gy) & \\
\hline \multirow[t]{2}{*}{ Higuchi et al [27] } & 2014 & Japan & Retrospective & $42 / 0$ & I-IV & Standard dose & 30 & $50.4 \mathrm{~Gy}$ & 3D-CRT and IMRT \\
\hline & & & & & & High dose & 12 & $61.2 \mathrm{~Gy}$ & \\
\hline \multirow[t]{2}{*}{ Nemoto et al [28] } & 2020 & Japan & Retrospective & $951 / 17$ & I-IV & Standard dose & 171 & $50.4 \mathrm{~Gy}$ & 3D-CRT and IMRT \\
\hline & & & & & & High dose & 825 & 60Gy & \\
\hline
\end{tabular}

SCC, squamous cell carcinoma; AC, adenocarcinoma;

3D-CRT, 3-Dimensional conformal radiotherapy; IMRT, Intensity-modulated radiation therapy;

\# Median radiation dose and the dose range of the whole group.

*Construct a propensity score matched cohort (1:1 for high dose vs standard dose)

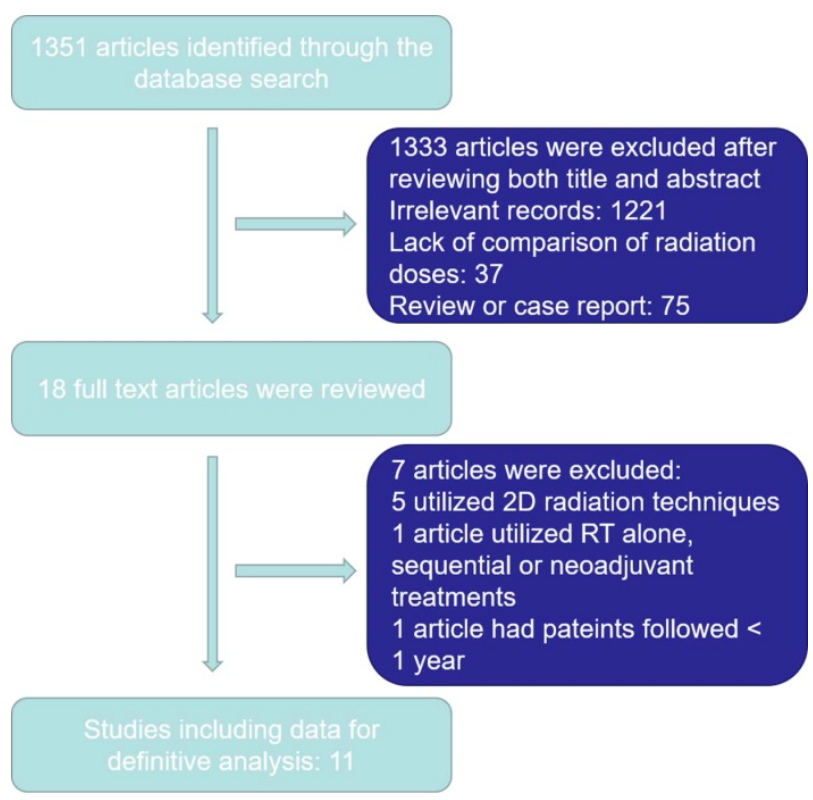

Figure 1. Study selection process.

\section{Outcome data}

Five studies analyzed $\mathrm{CCR}$ rates of the two groups $[17,18,23,27,28]$. There was no difference between the two groups in this respect (OR 0.862, 95\% CI 0.406-1.829; $\mathrm{P}=0.698$, Figure 2).

Three articles analyzed the LRF rate of the two groups [14, 17, 25]. High-dose group had a significant advantage over the standard-dose group in this respect (OR 2.199, 95\% CI 1.487-3.253; P<0.001, Figure $3)$.

Two articles analyzed two-year LRC rates of the two groups $[17,25]$. High-dose group had a significant advantage over the standard-dose group (OR 0.478, 95\% CI 0.309-0.740; P=0.001, Figure 4).

Five studies analyzed the two-year OS of the two groups $[16,18,19,23,26]$. High-dose group had a significant advantage over the standard-dose group (HR 0.744, 95\% CI 0.657-0.843; P<0.001, Figure 5).

Five studies analyzed the five-year OS of the two groups $[14,15,17,19,26]$. High-dose group had a significant advantage over the standard-dose group (HR 0.683, 95\% CI 0.561-0.831; P<0.001, Figure 6).

Five articles analyzed grade $\geq 3$ radiation-related toxicities, with treatment-related deaths evaluated in the four series $[14,17,19,23,27]$ (Table 2). No difference was seen between the two groups in treatment-related deaths (OR 1.026, 95\% CI 0.353-2.982; $\mathrm{P}=0.963$ ), radiation-related esophagitis (OR 0.668, 95\% CI 0.385-1.159; $\mathrm{P}=0.152$ ), pneumonitis (OR 2.654, 95\% CI 0.830-8.480; $\mathrm{P}=0.1$ ), esophageal stenosis (OR 0.578, 95\% CI 0.316-1.060; $\mathrm{P}=0.076$ ) and esophageal fistula (OR 0.927, 95\% CI 0.277-3.103; $\mathrm{P}=0.903$ ).

\section{Sensitivity analysis}

Sensitivity analysis showed that the new combined HR of two-year OS rate were different from the original HR while other items were similar to the original HR/OR (Table 3).

\section{Publication bias analysis}

Funnel plot was used to evaluated the publication bias. Egger's regression test was conducted to analyze the symmetry of the funnel plot (Table 4). None of the articles demonstrated publication bias $(\mathrm{P}>0.05)$. 


\section{Study}

$\%$

Weight

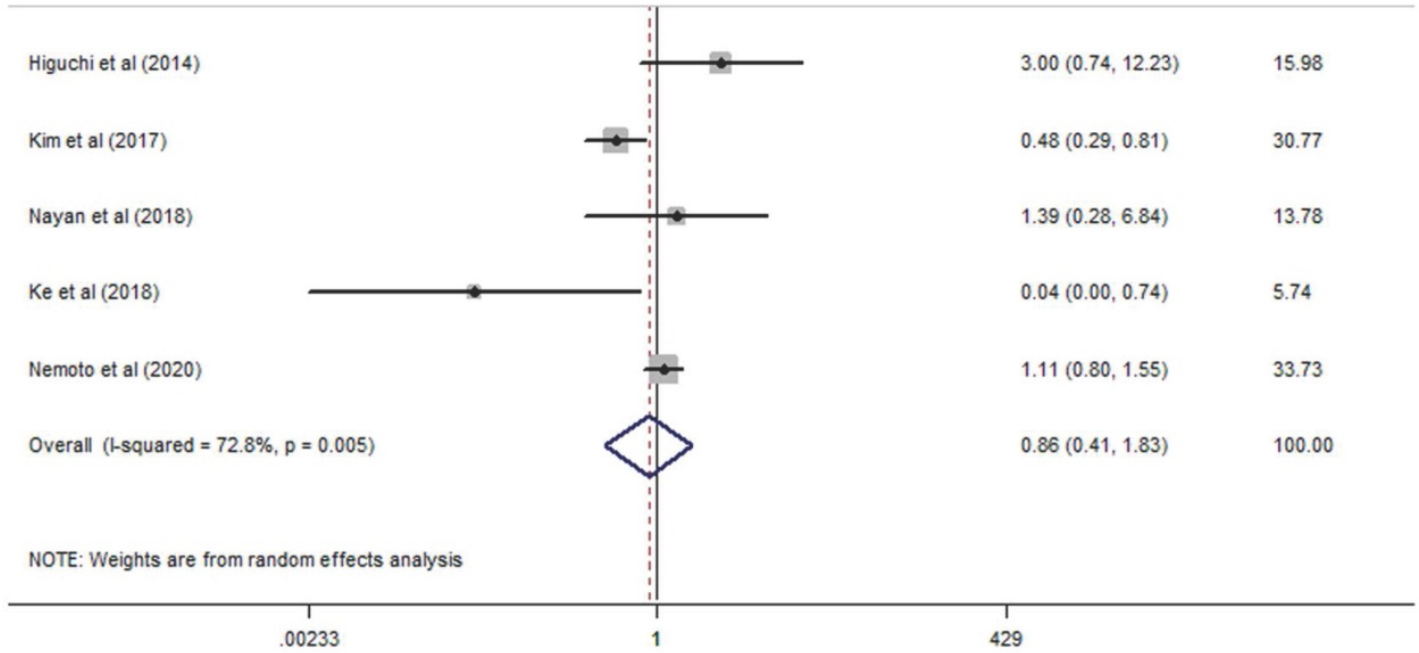

High-dose group

Standard-dose group

Figure 2. Effects of high- and standard-doses on $\mathrm{CCR}$ rate. $\mathrm{Cl}$, confidence interval; OR, odds ratio.

$$
\text { Study }
$$

ID

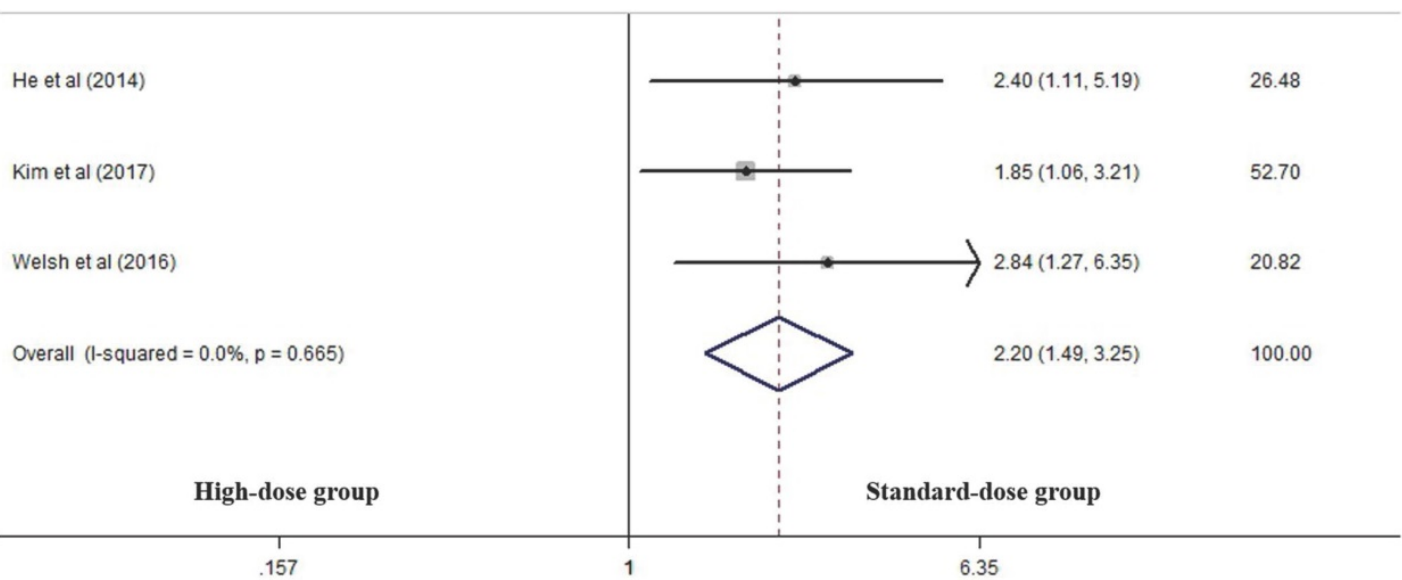

$\%$

OR $(95 \% \mathrm{Cl}) \quad$ Weight

Figure 3. Effects of high- vs. standard-dose RT on local recurrence rate. $\mathrm{Cl}$, confidence interval; OR, odds ratio.

Table 2. Grade $\geq 3$ radiation-related toxicities and treatment-related deaths

\begin{tabular}{|c|c|c|c|c|c|}
\hline Article & Treatment-related death" & Esophagitis" & Pneumonitis" & Esophageal stenosis" & Esophageal fistula" \\
\hline He et al [14] & $5.1 \% / 3.6 \%$ & $20.4 \% / 17.9 \%$ & $6.6 \% / 0 \%$ & $18.3 \% / 32.1 \%$ & $2.2 \% / 3.6 \%$ \\
\hline Kim et al [17] & $1.7 \% / 0.9 \%$ & -- & $1.7 \% / 0 \%$ & $5 \% / 5.2 \%$ & $1.7 \% / 1.7 \%$ \\
\hline Ren et al [19] & $0.5 \% / 1.6 \%$ & $2.6 \% / 7.4 \%$ & $2.1 \% / 2.6 \%$ & -- & -- \\
\hline Higuchi et al [27] & -- & $23.3 \% / 41.6 \%$ & -- & -- & $6.7 \% / 0 \%$ \\
\hline Ke et al ${ }^{[23]}$ & $0 \% / 0 \%$ & $0 \% / 0 \%$ & $0 \% / 0 \%$ & $0 \% / 0 \%$ & $0 \% / 0 \%$ \\
\hline
\end{tabular}

\# standard-dose group/high-dose group. 
Study

ID

Kim et al (2017)

Welsh et al (2016)

Overall (I-squared $=0.0 \%, p=0.697$ )

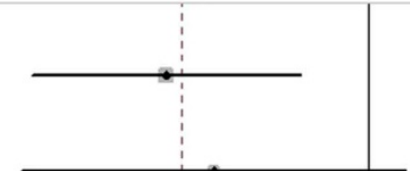

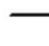

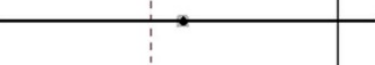

$0.54(0.25,1.16)$

30.75

$0.48(0.31,0.74) \quad 100.00$

\begin{tabular}{c:cc|c} 
& & \\
High-dose group & Standard-dose group \\
\hline .252 & & \\
\hline
\end{tabular}

Figure 4. Effects of high- vs. standard-dose RT on two-year local-regional control rate. Cl, confidence interval; OR, odds ratio.
Study
ES $(95 \% \mathrm{Cl}) \quad$ Weight

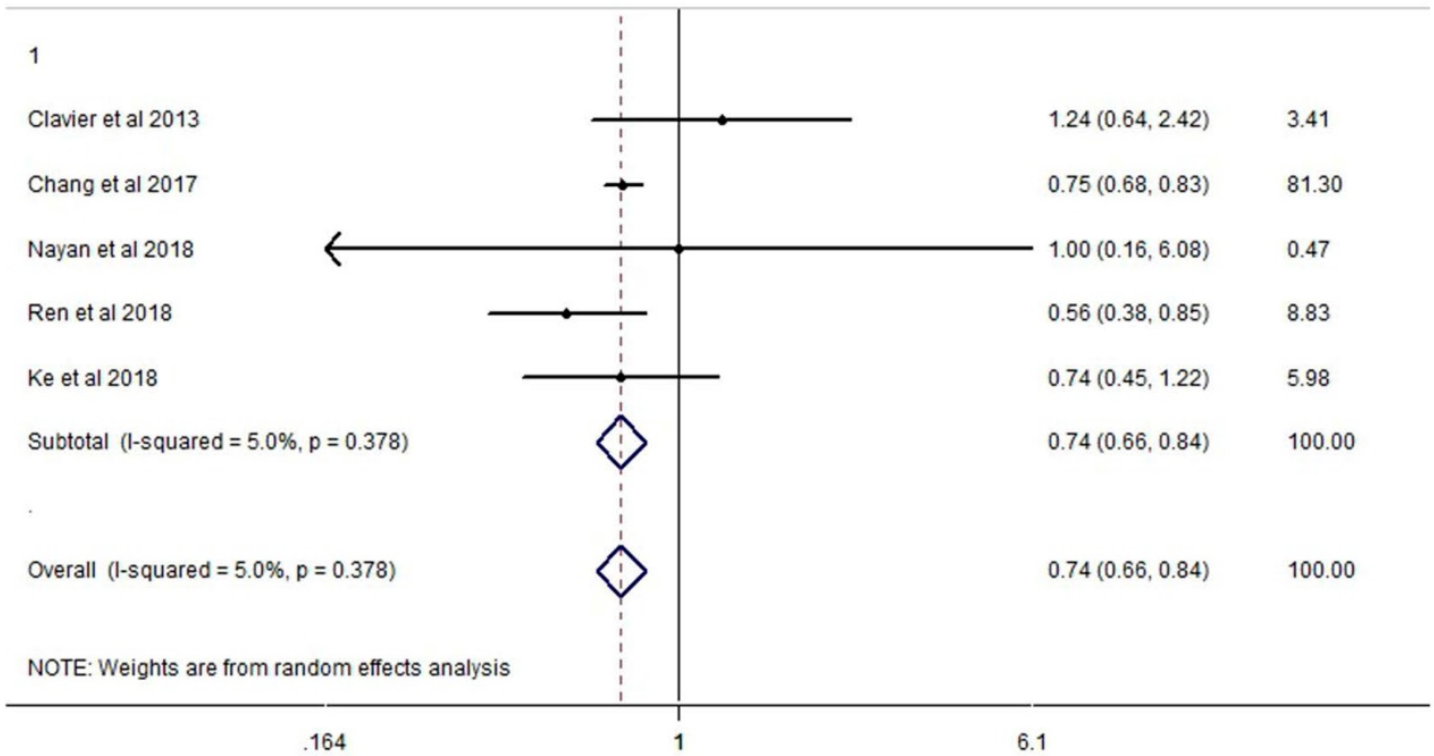

High-dose group

Standard-dose group

Figure 5. Effects of high- vs. standard-dose RT on two-year OS. Cl, confidence interval; ES, effect size.

Table 3. Sensitivity analysis

\begin{tabular}{|c|c|c|c|c|}
\hline Item & Deleted article & HR/OR & $95 \% \mathrm{CI}$ & $P$ \\
\hline cCR rate & Nemoto et al [28] & 0.734 & $0.206-2.617$ & 0.633 \\
\hline LRF rate & Kim et al [17] & 2.593 & $1.485-4.530$ & 0.001 \\
\hline Two-year LRC rate & -- & -- & -- & -- \\
\hline Two-year OS & Chang et al [16] & 0.748 & $0.523-1.968$ & 0.11 \\
\hline Five-year OS & Chen et al [15] & 0.591 & $0.444-0.787$ & $<0.001$ \\
\hline Treatment-related death & Ren et al [19] & 1.588 & $0.416-6.062$ & 0.499 \\
\hline Esophagitis & Ren et al [19] & 0.935 & $0.471-1.856$ & 0.847 \\
\hline Pneumonitis & Ren et al [19] & 6.874 & $0.847-55.762$ & 0.071 \\
\hline Esophageal stenosis & -- & -- & -- & -- \\
\hline Esophageal fistula & He et al [14] & 1.266 & $0.250-6.414$ & 0.776 \\
\hline
\end{tabular}

HR, hazard ratio; OR, odds ratio; CI, confidence interval; cCR clinical complete response; LRF, local-regional failure; OS, overall survival. 


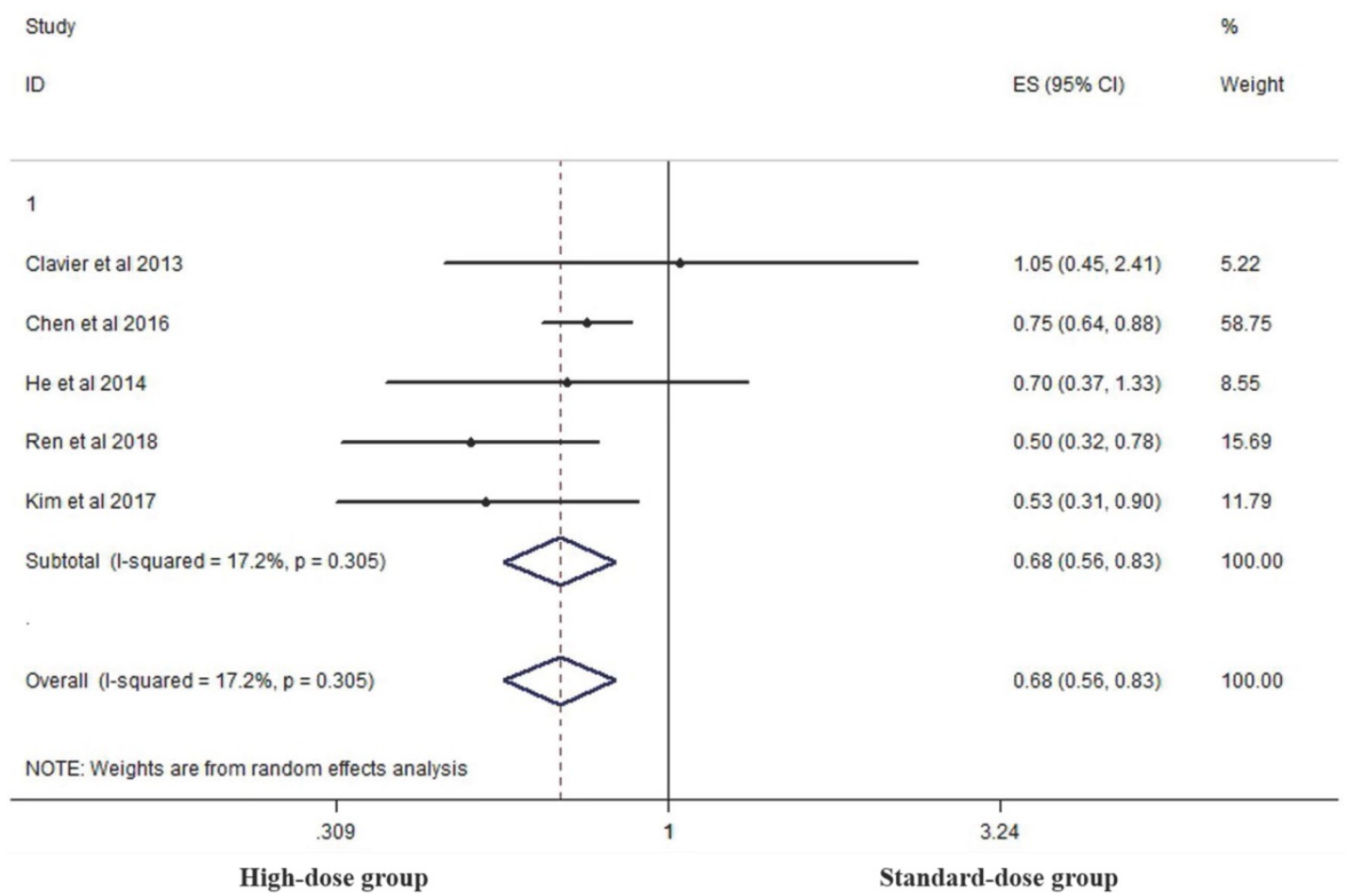

Figure 6. Effects of high- vs. standard-dose RT on five-year OS. $\mathrm{Cl}$, confidence interval; ES, effect size.

Table 4. Publication bias results of selected articles

\begin{tabular}{llll}
\hline Evaluation Items & $\mathbf{t}$ & $\mathbf{9 5 \%} \mathbf{C I}$ & $\boldsymbol{P}$ \\
\hline cCR rate & 0.25 & $-8.056-9.037$ & 0.828 \\
LRF rate & 4.53 & $-5.327-11.238$ & 0.138 \\
Two-year LRC rate & -- & - & -- \\
Two-year OS & 0.28 & $-2.113-2.520$ & 0.798 \\
Five-year OS & -0.68 & $-3.529-2.290$ & 0.546 \\
Treatment-related death & -0.37 & $-45.214-42.684$ & 0.777 \\
Esophagitis & -1.14 & $-46.51-38.834$ & 0.458 \\
Pneumonitis & 3.69 & $-6.556-11.931$ & 0.168 \\
Esophageal stenosis & -- & - & -- \\
Esophageal fistula & 2.84 & $-6.389-10.059$ & 0.216
\end{tabular}

$\mathrm{CI}$, confidence interval; cCR, clinical complete response; LRF, local-regional failure; OS, overall survival.

\section{Discussion}

This study demonstrated that a radiation dose of $\geq 60$ Gy utilizing CT-based radiation techniques for the dCCRT of esophageal cancer might decrease LRF and improve two-year LRC, and five-year OS rates of patients without increasing toxicity rates compared to standard RT doses. As there were approximately $97 \%$ of patients with ESCC in this study, whether this conclusion was applicable to patients with EAC requires further verification.

RTOG 85-01 and RTOG 94-05/INT 0123 trials established a dose of $50.4 \mathrm{~Gy}$ in the dCCRT for patients with inoperable, localized EC [4-6]. The RTOG 94-05 trial represents the only large randomized controlled trial (RCT) evaluating RT dose in this setting, comparing the effect of high-dose (64.8 Gy) versus standard-dose (50.4 Gy) treatment. Study results showed that the high-dose arm failed to demonstrate any survival benefit while showing a higher treatment-related mortality rate [6]. However, there were caveats, including that seven of the eleven treatment-related deaths in the high-dose group occurred before $50.4 \mathrm{~Gy}$, i.e. dose-escalation was not the cause of death in these patients. Additionally, patients in the high-dose group underwent a treatment break for side effects recovery, resulting in a significantly prolonged treatment time. Finally, patients in the high-dose group received significant lower doses of 5-fluorouracil (5-FU) compared to those in the standard-dose group. These and other factors have the potential to influence ultimate outcomes and potentially negate any benefits of dose-escalation. Additionally, radiation technique in this study was based on what are largely historical 2D approaches, as well as utilized fields larger than that used in contemporary practice, which may increase toxicity rates and not be well suited for dose-escalation.

More recently, improvements in radiotherapy techniques (including CT-based planning), have led to reevaluation of dose-escalation approaches in the dCCRT of esophageal cancer. In a study by Suh et al [21], the high-dose group showed significant improvement in 2-year LRC rate $(69 \%$ vs. $32 \%$, $\mathrm{P}<0.01)$. Ren and colleagues found that the 10-year LRC rate increased by more than $20 \%$ ( $52 \%$ vs. $29.8 \%$, 
$\mathrm{P}=0.028$ ) with higher radiation doses [19]. Similarly, Kim et al demonstrated that their high-dose group experienced significantly lower LRF-alone rates ( $25.9 \%$ vs. $39.2 \%, \mathrm{P}=0.029)$ and a significantly higher 2-year LRC rate $(69.1 \%$ vs. $50.3 \%$, p $=0.002)$ relative to the standard-dose group [17]. He et al also showed that their high-dose group had a significantly lower LRF rate $(17.9 \%$ vs. $34.3 \%, \mathrm{P}=0.024)$ [14]. Welsh et al. use simultaneous integrated boost (SIB)-IMRT technique to dose-escalate gross disease and demonstrated that this approach could reduce LRF rates for patients with unresectable locally advanced EC (29\% vs. $54 \%$, $\mathrm{P}=0.01$ ) [25]. Along these lines, the current meta-analysis also demonstrated that the high-dose group had significantly lower LRF rates (OR 2.199, 95\% CI 1.487-3.253; P<0.001) and higher two-year LRC rates (OR 0.478, 95\% CI 0.309-0.740; $\mathrm{P}=0.001$ ) compared to the standard-dose group.

Given the above, the role of increased LRC on survival benefits has also been explored. Some studies have indicated that higher radiation dose may lead improved survival for ESCC patients undergoing dCCRT [15-17, 19-21, 23-24], while others have not [14, 18, 26-30]. A recent registry study reported by Japanese investigators demonstrated 5-year OS rates for stage groups I, II/III and IV were $64.2 \% / 57.2 \%$, $35.0 \% / 27.0 \%$ and $18.0 \% / 15.3 \%$ in the $50.4 \mathrm{~Gy}$ and 60 Gy groups, respectively, with no superiority of the high-dose group in any stage group [28]. A meta-analyses by Song et al in 2015 included 55 articles and concluded that high-dose radiation increased treatment response and 5-year OS rates while decreasing LRF and distant failure rates with without additional toxicity, notably for ESCC patients [31]. Another study by Chen et al analyzed 18 articles and also found that a dose of $\geq 60$ Gy appeared to improve OS and LRC, notably in Asian patients [32]. Luo et al. reached a similar conclusion [33]. In spite of this, the studies analyzed in these three meta-analyses included many series implementing 2D radiation techniques, which may not reflect the real impact of dose-escalation in modern practice and planning. In the current study, we defined inclusion criteria to include only studies utilizing CT-based radiation planning techniques. Our results demonstrated that a dose of $\geq 60$ Gy appeared to increase 5-year OS compared to standard doses, without increasing treatment related toxicities, supporting this strategy in the modern planning approaches in these patients. Based on the current data, the chemotherapy regimens used were not significantly different between the two groups, respectively, in most enrolled studies. While as the limitation of the data, we cannot analyze the effects of the different chemotherapy regimens. As previous researches reported [34,35], different chemotherapy regimens in the definitive chemoradiotherapy may not affect the survival of patients.

In view of the above results, it is important to review the underlying principles of dose escalation. As reported by Fletcher over four decades ago [36], biologically, doses of 45-50 Gy are generally adequate to control microscopic disease, $\geq 60$ Gy required to control gross disease, and nearly 100 Gy to cure solid tumors. In other diseases, including non-small cell lung cancer, it has been estimated that there is an approximate $1 \%$ improvement in long-term LRC and $3 \%$ decrease in the hazard from death with each additional Gy of radiation delivered [37]. To our knowledge, the failure of RTOG 94-05 was related to high rates of toxicities in $2 \mathrm{D}$ era. A latest study in Japan used proton beam therapy for esophageal cancer and reported lower rates of toxicities in comparison to photon radiotherapy [38]. The biological effective dose (BED) in this study was up to 87.2 Gy (67.2 - 96.1 Gy). The 3-year, 5-year OS rate and five-year LC rate was $66.7 \%, 56.3 \%$ and $64.4 \%$, respectively. The 5-year OS rate based on stage IV were $28.3 \%$. 3D radiotherapy techniques could control the toxicities and side effects, so higher doses may bring survival benefits to patients. In most Asian countries, where ESCC is the predominant histological type, doses of $\geq 60$ Gy are much common. Exemplifying this, in the current meta-analysis, ESCC was the predominant histology (approximately 96.5\% of patients) and eight of eleven articles included were from Asian countries/only two from the west, making our results particularly relevant to patients with ESCC from Asian countries.

There are limits to this meta-analysis. Firstly, articles were searched among published articles written in English and statistical analysis was limited to the published data. So, publication and language bias maybe exist. Secondly, ten of eleven included studies were retrospective studies (with the inherit limitations), with the one prospective randomized study only included 28 cases. It was a pity that no randomized trial and prospective study with large cases have been published yet (trials ongoing). But two studies with large cases (Chen et al [15] and Ren et al [19]) constructed a propensity score matched cohort to balance observable potential confounders. Additional RCTs are needed to verify our conclusion, with many ongoing (Table 5). Thirdly, our data include that the combined HR/OR of two-year OS rate were different from the original results following sensitivity analysis. Additionally, we did not perform subgroup analyses with regard to tumor site, stage and pathological type, with locally advanced, thoracic ESCC tumors comprising most of our population. 
Finally, although CCR is defined as not visible tumor following dCCRT [39], in the clinical practice, specific evaluation methods and criterion of $\mathrm{CCR}$ may not be uniform, creating uncertainty as to whether $\mathrm{CCR}$ following dCCRT is able to guide subsequent treatment recommendations and requires further study. These factors might have influenced our findings and conclusion.

In conclusion, our study demonstrated that, compared to the standard radiation doses, dose-escalated ( $\geq 60$ Gy), CT-based radiotherapy techniques might improve ultimate disease-related outcomes in patients with inoperable ESCC under the premise of controlling toxicity rates, and represents the first meta-analysis comparing high- versus standard-radiation doses utilizing CT-based/modern radiotherapy techniques. While our review supports a dose-escalation approach in these patients, multiple ongoing randomized trial initial and final reports are awaited to evaluate the effectiveness of this strategy. In addition, as there were approximately $97 \%$ of patients with ESCC in this study, whether this conclusion was applicable to patients with EAC similarly requires further verification.

Table 5. Selected ongoing RCTs evaluating dose escalation

\begin{tabular}{|c|c|c|c|c|c|}
\hline NCT number & Country & $\begin{array}{l}\text { Start } \\
\text { Date }\end{array}$ & $\begin{array}{l}\text { Estimated } \\
\text { Completion } \\
\text { Date }\end{array}$ & Groups & $\begin{array}{l}\text { Estimated } \\
\text { Enrollment }\end{array}$ \\
\hline NCT 01348217 & France & 2011 & 2019 & 66 Gy vs. 50 Gy & 252 \\
\hline NCT 01937208 & China & 2013 & 2017 & 60 Gy vs. 50 Gy & 300 \\
\hline NCT 02556762 & China & 2015 & 2021 & 66/50 Gy (SIB) vs. 50 Gy & 202 \\
\hline NCT 02741856 & UK & 2016 & 2023 & 60 Gy vs. 50 Gy & 584 \\
\hline NCT 02850991 & China & 2016 & 2021 & 59.4 Gy vs. 50.4 Gy & 308 \\
\hline NCT 03790553 & China & 2018 & 2025 & 61.2 Gy vs. 50.4 Gy & 646 \\
\hline
\end{tabular}

\section{Acknowledgements}

This study was funded by the Clinical Translational Research of Achievement in Tumor Field (162777114D), the Preoperative Concurrent Chemoradiotherapy for Potentially Resectable Adenocarcinoma of the Esophagogastric Junction (A-C) (NCT01962246), the 2016 Beijing Hope Marathon Fund of China Cancer Foundation (LC2016W10), Medical Science Research Key Projects of Hebei Provincial Department (ZD20140060), and Government Funding for Clinical and Scientific Research Capacity Building by Hebei Provincial Department of Finance (361006).

\section{Competing Interests}

The authors have declared that no competing interest exists.

\section{References}

1. Bray F, Ferlay J, Soerjomataram I, et al. Global cancer statistics 2018: GLOBOCAN estimates of incidence and mortality worldwide for 36 cancers in 185 countries. CA Cancer J Clin. 2018; 68(6): 394-424.

2. Siegel RL, Miller KD, Jemal A. Cancer statistics, 2019. CA Cancer J Clin. 2019; 69(1): 7-34.

3. National Comprehensive Cancer Network guidelines for esophageal cancer, version 2. 2019. Available at: https://www.nccn.org/professionals/ physician_gls/pdf/esophageal.pdf. Accessed May 29, 2019.

4. Herskovic A, Martz K, Al-Sarraf M, et al. Combined chemotherapy and radiotherapy compared with radiotherapy alone in patients with cancer of the esophagus. N Engl J Med. 1992; 326(24): 1593-1598.

5. Cooper JS, Guo MD, Herskovic A, et al. Chemoradiotherapy of locally advanced esophageal cancer: long-term follow-up of a prospective randomized trial (RTOG 85-01). Radiation Therapy Oncology Group. JAMA. 1999; 281(17): 1623-1627.

6. Minsky BD, Pajak TF, Ginsberg RJ, et al. INT 0123 (Radiation Therapy Oncology Group 94-05) phase III trial of combined-modality therapy for esophageal cancer: high-dose versus standard-dose radiation therapy. J Clin Oncol. 2002; 20(5): 1167-1174

7. Rohatgi PR, Swisher SG, Correa AM, et al. Failure patterns correlate with the proportion of residual carcinoma after preoperative chemoradiotherapy for carcinoma of the esophagus. Cancer. 2005; 104(7): 1349-1355.

8. Rawat S, Kumar G, Kakria A, et al. Chemoradiotherapy in the management of locally advanced squamous cell carcinoma esophagus: is surgical resection required? J Gastrointest Cancer. 2013; 44(3): 277-284.

9. Kawaguchi $Y$, Nishiyama K, Miyagi K, et al. Patterns of failure associated with involved field radiotherapy in patients with clinical stage I thoracic esophageal cancer. Jpn J Clin Oncol. 2011; 41(8): 1007-1012.

10. van Hagen $\mathrm{P}$, Hulshof $\mathrm{MC}$, van Lanschot JJ, et al. Preoperative chemoradiotherapy for esophageal or junctional cancer. N Engl J Med. 2012; 366(22): 2074-2084

11. Kato K, Nakajima TE, Ito $\mathrm{Y}$, et al. Phase II study of concurrent chemoradiotherapy at the dose of 50.4 Gy with elective nodal irradiation for Stage II-III esophageal carcinoma. Jpn J Clin Oncol. 2013; 43(6): 608-615.

12. Xi M, Yang Y, Zhang L, et al. Multi-institutional analysis of recurrence and survival after neoadjuvant chemoradiotherapy of esophageal cancer: impact of histology on recurrence patterns and outcomes. Ann Surg. 2018; 269(4): 663-670.

13. Xi M, Xu C, Liao Z, et al. The impact of histology on recurrence patterns in esophageal cancer treated with definitive chemoradiotherapy. Radiother Oncol. 2017; 124(2): 318-324.

14. He L, Allen PK, Potter A, et al. Re-evaluating the optimal radiation dose for definitive chemoradiotherapy for esophageal squamous cell carcinoma. J Thorac Oncol. 2014; 9(9): 1398-1405.

15. Chen $\mathrm{CY}, \mathrm{Li}$ CC, Chien CR. Does higher radiation dose lead to better outcome for non-operated localized esophageal squamous cell carcinoma patients who received concurrent chemoradiotherapy? A population-based propensity-score matched analysis. Radiother Oncol. 2016; 120(1): 136-139.

16. Chang CL, Tsai HC, Lin WC, et al. Dose escalation intensity-modulated radiotherapy-based concurrent chemoradiotherapy is effective for advanced-stage thoracic esophageal squamous cell carcinoma. Radiother Oncol. 2017; 125(1): 73-79.

17. Kim HJ, Suh YG, Lee YC, et al. Dose-response relationship between radiation dose and loco-regional control in patients with stage II-III esophageal cancer treated with definitive chemoradiotherapy. Cancer Res Treat. 2017; 49(3): 669-677.

18. Nayan N, Bhattacharyya M, Jagtap VK, et al. Standard-dose versus high-dose radiotherapy with concurrent chemotherapy in esophageal cancer: A prospective randomized study. South Asian J Cancer. 2018; 7(1): 27-30.

19. Ren X, Wang L, Han C, Ren L. Retrospective analysis of safety profile of high-dose concurrent chemoradiotherapy for patients with oesophageal squamous cell carcinoma. Radiother Oncol. 2018; 129(2): 293-299.

20. Zhang Z, Liao Z, Jin J, et al. Dose-response relationship in locoregional control for patients with stage II-III esophageal cancer treated with concurrent chemotherapy and radiotherapy. Int J Radiat Oncol Biol Phys. 2005; 61(3): 656-664

21. Suh YG, Lee IJ, Koom WS, et al. High-dose versus standard-dose radiotherapy with concurrent chemotherapy in stages II-III esophageal cancer. Jpn J Clin Oncol. 2014; 44(6): 534-540.

22. Deng $\mathrm{Y}$, Bian $\mathrm{C}$, Tao $\mathrm{H}$, et al. Improved survival with higher radiation dose for esophageal squamous cell carcinoma patients treated with definitive chemoradiotherapy. Oncotarget. 2017; 8(45): 79662-79669.

23. Ke TM, Fong Y, Lin LC, et al. Evaluating the optimal radiation dose for definitive chemoradiotherapy for esophageal squamous cell carcinoma: A single institution experience. Medicine (Baltimore). 2018; 97 (46): e13214.

24. Li CC, Fang HY, Lin CY, et al. Outcomes of localized esophageal squamous cell carcinoma patients treated with definitive concurrent chemoradiotherapy using either standard or high radiotherapy dose: A retrospective study controlling for organ at risk dose. Anticancer Res. 2019; 39 (1): 511-517.

25. Welsh JW, Seyedin SN, Allen PK, et al. Local control and toxicity of a simultaneous integrated boost for dose escalation in locally advanced esophageal cancer: Interim results from a prospective phase I/II trial. J Thorac Oncol. 2017; 12 (2): 375-382. 
26. Clavier JB, Antoni D, Atlani D, et al. Definitive chemoradiotherapy for esophageal cancer: 66Gy versus 50Gy, a retrospective study. Cancer Radiother. 2013; 17(3): 221-228.

27. Higuchi K, Komori S, Tanabe S, et al. Definitive chemoradiation therapy with docetaxel, cisplatin, and 5-fluorouracil (DCF-R) in advanced esophageal cancer: a phase 2 trial (KDOG 0501-P2). Int J Radiat Oncol Biol Phys. 2014; 89(4): 872-879.

28. Nemoto K, Kawashiro S, Toh Y, et al. Comparison of the effects of radiotherapy doses of $50.4 \mathrm{~Gy}$ and $60 \mathrm{~Gy}$ on outcomes of chemoradiotherapy for thoracic esophageal cancer: subgroup analysis based on the Comprehensive Registry of Esophageal Cancer in Japan from 2009 to 2011 by the Japan Esophageal Society. Esophagus 2020; [Epub ahead of print].

29. Brower JV, Chen S, Bassetti MF, et al. Radiation dose escalation in esophageal cancer revisited: A contemporary analysis of the National Cancer Data Base, 2004 to 2012. Int J Radiat Oncol Biol Phys. 2016; 96(5): 985-993.

30. De B, Rhome R, Doucette J, et al. Dose escalation of definitive radiation is not associated with improved survival for cervical esophageal cancer: A National Cancer Data Base (NCDB) analysis. Dis Esophagus. 2017; 30(4): 1-10.

31. Song $T$, Liang $X$, Fang $M$, et al. High-dose versus conventional-dose irradiation in cisplatin-based definitive concurrent chemoradiotherapy for esophageal cancer: a systematic review and pooled analysis. Expert Rev Anticancer Ther. 2015; 15(10): 1157-1169.

32. Chen $Y$, Zhu HP, Wang T, et al. What is the optimal radiation dose for non-operable esophageal cancer? Dissecting the evidence in a meta-analysis. Oncotarget. 2017; 8(51): 89095-89107.

33. Luo HS, Huang HC, Lin LX. Effect of modern high-dose versus standard-dose radiation in definitive concurrent chemo-radiotherapy on outcome of esophageal squamous cell cancer: a meta-analysis. Radiat Oncol. 2019; 14(1): 178.

34. Chen Y, Ye J, Zhu Z, et al. Comparing Paclitaxel Plus Fluorouracil Versus Cisplatin Plus Fluorouracil in Chemoradiotherapy for Locally Advanced Esophageal Squamous Cell Cancer: A Randomized, Multicenter, Phase III Clinical Trial. J Clin Oncol. 2019 Jul 10;37(20):1695-1703.

35. Liu Y, Ren Z, Yuan L, et al. Paclitaxel plus cisplatin vs. 5-fluorouracil plus cisplatin as first-line treatment for patients with advanced squamous cell esophageal cancer. Am J Cancer Res. 2016 Oct 1;6(10):2345-2350.

36. Fletcher GH. Clinical dose-response curves of human malignant epithelial tumors. Br J Radiol. 1973; 46(541): 1-12.

37. Kong FM, Ten Haken RK, Schipper MJ, et al. High-dose radiation improved local tumor control and overall survival in patients with inoperable/unresectable non-small-cell lung cancer: long-term results of a radiation dose escalation study. Int J Radiat Oncol Biol Phys. 2005; 63(2): 324-333.

38. Ono T, Wada H, Ishikawa H, et al. Clinical Results of Proton Beam Therapy for Esophageal Cancer: Multicenter Retrospective Study in Japan. Cancers (Basel). 2019; 11 (7).

39. Wang J, Qin J, Jing S, et al. Clinical complete response after chemoradiotherapy for carcinoma of thoracic esophagus: Is esophagectomy always necessary? A systematic review and meta-analysis. Thorac Cancer. 2018; 9(12): 1638-1647. 